\title{
Der «letzte Zufluchtsort für Stürmer und Dränger»? Psychoanalytische Pädagogik in den Berner Seminarblättern und der Schulreform 1907-1930
}

\section{Patrick Bühler}

An den reformpädagogischen Berner Seminarblättern und ihrer Weiterführung Die Schulreform, zu deren Herausgebern die psychoanalytischen Pädagogen Ernst Schneider und Oskar Pfister zählen, lässt sich eine erstaunlich frühe und bemerkenswert positive pädagogische Psychoanalyse-Rezeption und deren reformpädagogischer Stellenwert studieren. Wie die Untersuchung zeigt, wird Psychoanalyse in der Schweizer Zeitschrift allerdings äusserst selektiv rezipiert und ist das reformpädagogische Interesse daran gering.

\section{Genf, Bern, Zürich}

Im Jahrbuch der Schweizerischen Gesellschaft für Schulgesundheitspflege erscheint 1914 ein Artikel mit der Überschrift «Les innovations les plus importantes du domaine de la pédagogie depuis le début du siècle». Der Genfer Psychologieprofessor und Gründer des Institut Jean-Jacques Rousseau Édouard Claparède unterscheidet darin wissenschaftliche von praktischen Neuerungen, zu denen er z. B. die Arbeitsschule, Freiluftschulen oder die Montessori-Methode zählt. Bei den wissenschaftlichen Fortschritten schafft es die "Psychanalyse (Autriche 1900)» auf den dritten Platz (Claparède, 1914, S. 248) ${ }^{1}$. Dass Claparède ausgerechnet der Psychoanalyse als einer der "wichtigsten pädagogischen Neuerungen» seit der Jahrhundertwende seine Ehrerbietung erweist, dürfte zumindest unter seinen Genfer Kollegen wenig Erstaunen hervorgerufen haben. Denn wie Claparède selbst betont, nimmt die Stadt an der Rhone in dieser Hinsicht eine Sonderstellung ein: "Genève, il faut le dire, avait fait exception, dans les pays de langue française, à ce mouvement général d'ostracisme ou d'indifférence» (Claparède, 1920, S. 850). So begeistert sich etwa auch Théodore Flournoy, der 1891 in Genf auf einen Lehrstuhl für Psychologie berufen wird, schon früh für diese neue Wissenschaft: «A peine parus, les premiers travaux de Freud étaient cités et commentés par M. Flournoy, dont la perspicacité avait vite saisi leur valeur» (Claparède, 1920, S. 850)2 . Aber nicht nur Flournoy und sein Cousin Claparède 
setzen sich mit Psychoanalyse auseinander, sondern auch andere Mitarbeiter des 1912 gegründeten Institut Jean-Jacques Rousseau. So tritt z. B. Pierre Bovet, der erste Leiter des Instituts, gleich der 1919 gegründeten Schweizerischen Gesellschaft für Psychoanalyse bei (Oberholzer, 1920, S. 107). Zudem ist er Mitglied der Groupe psychanalytique de Genève, die von Claparède geleitet wird (vgl. Roudinesco, 1986, S. 362-368), und veröffentlicht 1920 "La psychanalyse et l'éducation" (Bovet, 1920). Charles Baudouin, der 1915 am Institut zu arbeiten beginnt und dort überhaupt erst die Psychoanalyse kennen lernt, veröffentlicht 1922 z. B. seine Études de psychanalyse und 1931 L'àme enfantine et la psychanalyse. Beide Titel erscheinen in der "offiziellen" Publikationsreihe des Instituts (vgl. Cifali, 2005; Ruchat, 2005). Schliesslich hat sich auch Jean Piaget - zumindest eine Zeitlang - eingehend mit Psychoanalyse beschäftigt. 1918 unterzieht er sich in Zürich nicht nur einer ersten Kur, sondern behandelt auch selbst «mindestens fünf Analysanden» (Kohler, 2009, S. 52). 1920 tritt Piaget der Schweizerischen Gesellschaft für Psychoanalyse bei, in der Sitzung spricht Bovet passenderweise zu "L'inconscient à Genève» (Oberholzer, 1921, S. 129). Anfang der zwanziger Jahre beginnt Piaget in Genf eine Lehranalyse bei Sabina Spielrein, die auch die Analytikerin Claparèdes und Bovets ist. Piaget publiziert drei psychoanalytische Artikel (Piaget, 1993) und unternimmt sogar den am Ende erfolglosen «Versuch, seine eigene Mutter zu therapieren» (Kohler, 2009, S. 59) ${ }^{3}$.

Wenn in den zwanziger und dreissiger Jahren «weder in der romanischen Schweiz noch in Frankreich eine sogenannte psychoanalytische Pädagogik» entsteht (Cifali/Moll, 1995, S. 63), dann dürfte das daher nicht allein der nur zögerlich zu Stande kommenden Übersetzungen von Sigmund Freuds Werken oder gewissen Vorbehalten geschuldet sein, sondern auch an Genf liegen. Denn am Anfang nimmt die französische Psychoanalyse-Rezeption ihren Weg über die Schweiz und besonders über Genf (Ohayon, 2006, S. 82) und in diesem einflussreichen reformpädagogischen Zentrum ist Psychoanalyse eben Teil der pädagogischen Psychologie. Man braucht daher eine eigene psychoanalytische Pädagogik gar nicht erst zu entwickeln:

Il n'y a jamais eu chez nous les conflits entre la psychanalyse et la "psychologie officielle» ou expérimentale, qui se sont produits en tant de centres universitaires, et ont été aussi nuisibles au développement des recherches sur les phénomènes inconscients qu'à celui des travaux sur les fonctions supérieures de la vie mentale (Piaget, 1944, S. 100)

Im deutschen Sprachraum verläuft die pädagogische Psychoanalyse-Rezeption hingegen gerade "umgekehrt». Dem bekannten Jugendforscher William Stern käme es etwa nicht in den Sinn, wie Claparède die Psychoanalyse als «un des événements les plus importants qu'ait jamais eu enregistrer l'histoire de la science de l'esprit» zu feiern (Claparède, 1920, S. 864). Vielmehr veröffentlicht Stern 1913 einen scharfen «Protest» gegen «Die Anwendung der Psychoanalyse auf Kindheit und Jugend». Stern ist - wie viele andere - davon überzeugt, 
dass besonders «schwere Schädigung[en]» durch das völlig unwissenschaftliche, «magisch-mystische[] System der Zeichendeuterei» der Psychoanalyse entstünden, das allein im Dienst des «allseitigen Wittern[s] von Sexuellem» stehe. Gerade diese «Hypertrophie der Sexualgedanken» führe zu einer grauenhaften «Verzerrung und Verödung des Seelenbildes» und dazu, dass die Psychoanalyse "sich an der werdenden Seele» vergreife (Stern, 1913/1914, S. 73, 74, 77). Auch die beiden berühmten Wiener Forscher Charlotte und Karl Bühler halten wenig von Psychoanalyse (vgl. Benetka, 1995, S. 165-173, 246-249), als «Erziehungsmethode» sei sie «verhängnisvoll» (Bühler, 1922/1923, S. 127.). Insgesamt wird Psychoanalyse zu Beginn des 20. Jahrhunderts in der deutschen akademischen Pädagogik zwiespältig rezipiert. Man zollt der «anregenden» Theorie zwar Respekt, warnt aber vor den schlimmen Folgen ihrer Anwendung in der Pädagogik (vgl. Bühler, 2011, S. 34-40).

Die deutsche Schweiz und besonders Bern und Zürich sind nun ähnlich wie Genf eine aufschlussreiche "exception" (Claparède, 1920, S. 850), denn in diesen beiden Städten wird ebenso früh wie entschieden versucht, eine psychoanalytische Pädagogik zu entwickeln. Als besonders umtriebig erweisen sich der Berner Seminardirektor Ernst Schneider (1878-1957) und der Zürcher Pfarrer Oskar Pfister (1873-1956). Schneider beginnt sich um 1910 für Psychoanalyse zu interessieren und verliert 1916 unter anderem deswegen seine Stelle als Seminardirektor. Die Entlassung tut Schneiders pädagogisch-psychoanalytischem Engagement jedoch keinen Abbruch. Er arbeitet als Psychotherapeut, gründet z. B. 1926 zusammen mit Heinrich Meng die Zeitschrift für psychoanalytische Pädagogik und veröffentlicht 1930 die Abhandlung Psychoanalyse und Pädagogik5. Auch Pfister beschäftigt sich schon sehr früh mit Psychoanalyse. Es ist Carl Gustav Jung, der Pfister «in diese Probleme und ihre Behandlung ein[ ] weiht» (Pfister, 1909b, S. 139). Pfister macht sich auch früh daran, die Psychoanalyse zu popularisieren und auf die Pädagogik anzuwenden. Freud attestiert dem Pfarrer charmant, die "Anwendung der Analyse» «auf die Pädagogik» überhaupt «inauguriert» zu haben (Freud/Pfister, 1963, S. 112). Schon 1909 liefert Pfister sich etwa mit Friedrich Wilhelm Foerster, dem bekannten Verfasser einer Lebenskunde und einer Jugendlehre, einen öffentlichen Schlagabtausch über den Wert der Psychoanalyse (vgl. Pfister, 1909b; Pfister 1910; Foerster 1909; Foerster 1910) ${ }^{6}$. Dem Pfarrer kommt zweifelsohne eine «rôle central» «dans la diffusion de la psychanalyse dans le milieu pastoral et le monde pédagogique» zu (Moll, 1989, S. 55). Pfisters Renommee lässt sich etwa auch daran ablesen, dass er 1927 in die zweibändige Sammlung Die Pädagogik der Gegenwart in Selbstdarstellungen aufgenommen wird (Pfister, 1927).

Die reformpädagogischen Berner Seminarblätter und ihre Weiterführung Die Schulreform, zu deren Herausgebern Schneider und Pfister zählen, sind nun ein zweifach ausschlussreiches «Symptom». An der Schweizer Zeitschrift lässt sich nämlich sowohl eine erstaunlich frühe und bemerkenswert positive pädagogischen Psychoanalyse-Rezeption studieren als auch deren reformpädagogischer Stellenwert untersuchen. 


\section{Psychoanalyse und Reformpädagogik}

Im April 1907 erscheint die erste Nummer der Berner Seminarblätter, die von Schneider gegründet werden. Sie «entspringen dem Bedürfnis des Herausgebers» - wie Schneider «Zur Einführung» ausführt - «mit seinen Schülern nach der Seminarzeit in geistigem Kontakt zu bleiben, um ihnen bei ihrer Fortbildung im Dienste der pädagogischen Seite der Berufsarbeit behilflich zu sein». Dass dieser "geistige[] Kontakt» eher einseitig ausfallen dürfte, liegt in der Natur der Sache. Dass Schneider die Seminarblätter als «Ratgeber und Wegweiser» versteht, zeigt auch das «Titelbild» des Berner Künstlers Rudolf Münger: «Ein Jüngling

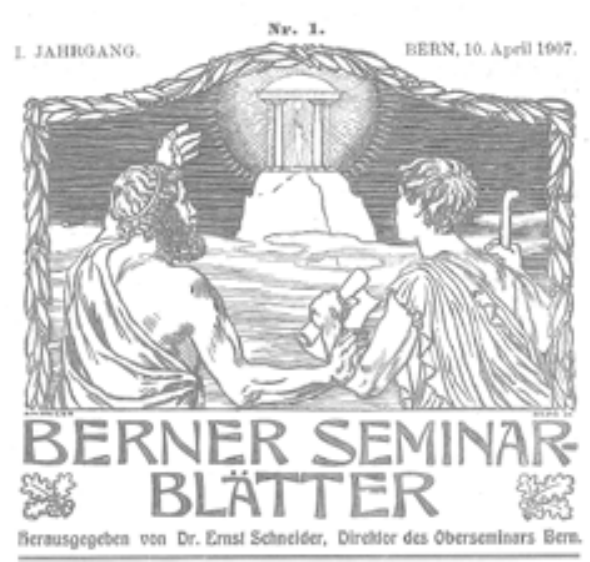
strebt, angezogen von einer Leuchte, dem Tempel der Wahrheit, der Schönheit und der Tugend zu. Auf dem Wege dazu bietet sich ihm ein Führer an». Das Programm, das Schneider in der ersten Nummer skizziert, umfasst «die Besprechung, Beurteilung und Würdigung der gegenwärtigen Bewegungen auf dem Gebiete des Bildungswesens", «[b]esondere Aufmerksamkeit wird [dabei] dem Fortschritt der expe-rimentellen Psychologie und Pädagogik und den Bestrebungen auf dem Gebiete der Kinderforschung gewidmet» (Schneider, 1907/1908, S. 1-2). Mit diesen "gegenwärtigen Bewegungen» und «Bestrebungen» sind wie auch das «Titelbild» verrät - reformpädagogische gemeint. So enthält z. B. der erste Jahrgang Beiträge zu "Vorstellungstypen», «Kinderfest[en]», «neue[n] schweizerische[n] Landerziehungsheim[en]», zur "Methode Jacques-Dalcroze», «Erziehung durch Kunst» oder «Waldschule». Ab 1910 schmückt der Untertitel «Monatsschrift für Schulreform» die Seminarblätter, ab 1916 heisst die Zeitschrift nur noch Die Schulreform. «Der Name ist ihr [der Zeitschrift] Programm»: «Es ist heute nötiger denn je, daß die Freunde der Schulreform zusammenstehen. Unterstützt unser Organ durch Abonnement und fleißige Lektüre!» (Anonym, 1916/1917, S. 1).

Mit Pfister zählt von 1912 bis 1926 neben Schneider ein weiterer prominenter psychoanalytischer Pädagoge zu den Herausgebern. Pfister ist - wie Schneider «Zum VI. Jahrgang» festhält - nicht nur «in das innere Wesen der Reformbewegung eingedrungen», sondern eben vor allem bekannt «als Vorkämpfer für eine pädagogische Verwertung der Freudschen Psychoanalyse» ([Schneider], $1912 / 1913,1)^{7}$. Will man die Psychoanalyse-Rezeption der Seminarblätter und der Schulreform abschätzen, bietet es sich an, die unter «Aufsätze» publizierten Beiträge auszuwerten, in deren Titel Termini wie "Freud», "Analyse» 
oder «Psychoanalyse» vorkommen und die gleichsam eine psychoanalytische Ausrichtung leicht erkennbar «vor sich her tragen». In den meisten Jahrgängen finden sich ab 1911 ein bis zwei solcher Artikel, insgesamt sind es 23 Beiträge. Ihr Anteil an allen Artikeln ist nicht sehr gross, er macht rund 3\% aus. Einsamer Spitzenreiter als Autor ist Pfister, der zwischen 1913 und 1919 sieben Artikel beisteuert. Der Sekundarlehrer und Psychotherapeut Gustav Hans Graber, der bei Schneider das Seminar besucht und von ihm analysiert wird (vgl. Schröter, 2000), erreicht mit nur drei Artikeln schon den zweiten Platz.

\section{"Wir haben viel von der Psychanalyse zu Lernen»}

Der Rorschacher Seminarlehrer Oskar Messmer veröffentlicht 1911 den ersten psychoanalytischen Beitrag, der in den Seminarblättern erscheint. Messmers «Die Psychanalyse und ihre pädagogische Bedeutung» endet mit der Feststellung8:

Alles in allem: Wir haben viel von der Psychanalyse zu lernen. Mit etwelcher Beschämung (in Ansehung unserer Versäumnis) aber auch mit tiefer Freude (in Ansehung des objektiven Fortschrittes) stellen wir fest, was für grosse und schöne Früchte uns von fremder Seite her in den pädagogischen Garten wachsen (Messmer, 1911/1912, S. 291-292).

Im September 1912 erscheint Messmers umfangreiche zweite und letzte psychoanalytische Arbeit «Die Psychanalyse in ihrer Entwicklung». Seine Studie belegt, dass es Messmer mit dem Lernen "von der Psychanalyse» durchaus ernst ist. Als er seinen ersten Artikel schreibt, kennt Messmer nämlich Freuds Traumdeutung noch gar nicht (Messmer, 1912/1913, S. 402, Fn.). Dieses «Versäumnis» hat er nun aufgeholt und sich in die "psychanalytische Literatur» vertieft (Messmer, 1912/1913, S. 520). So referiert Messmer in seinem zweiten Beitrag ausführlich und wohlwollend zahlreiche Arbeiten Freuds und seiner Schüler (z. B. Karl Abrahams, Jungs, Ludwig Binswangers, Otto Ranks). Messmer zufolge rührt die «hohe pädagogische Bedeutung der Psychanalyse» schlicht daher, dass es sich bei der Psychoanalyse um ein «Heilmittel» handle, dass «der Komplexwucherung entgegentritt und sie auflöst» (Messmer, 1912/1913, S. 489). Mit Messmers detaillierter Übersicht hat die Auseinandersetzung mit psychoanalytischen Theorien in den Berner Seminarblättern schon 1912/1913 ihren Zenit erreicht. Eine ähnlich einführende Darstellung versucht nur noch Pfister in «Psychanalyse und Jugendforschung» zu geben, es handelt sich aber nur um «ein kurzes Referat», das «sich auf spärliche Andeutungen beschränk[t]» (Pfister, 1914/1915b, S. 192-193) und fast ohne Verweise auf Sekundärliteratur auskommt.

Neben der Darstellung der psychoanalytischen Theorie beschäftigen sich eine Reihe weiterer Artikel mit der Psychoanalyserezeption und verschiedenen Detailfragen. Die Themen reichen von der Hinwendung des verstor- 
benen Berner Professors für Philosophie, Psychologie und Pädagogik Ernst Dürr zur Psychoanalyse (Pfister, 1913/1914a, S. 289) über Kritiken und Kritiker wie Foerster (Grüninger, 1921/1922a; Grüninger 1921/1922b; Pfister, 1918/1919a) bis zur Erörterung der umstrittenen Frage von «Psychoanalyse und christliche[m] Glaube[n]» (Blum 1921/1922; Burri, 1928/1929). Auch eine Gegenüberstellung von Freud und Oswald Spengler (Graber, 1923/1924) wird veröffentlicht ebenso wie ein Abdruck von Eugen Bleulers Gratulation zu Freuds 70. Geburtstag aus der Neuen Zürcher Zeitung (Bleuler, 1926/1927), ein Referat eines Artikels aus der Internationalen Zeitschrift für Psychoanalyse zur Selbstanalyse (Schwarz, 1926/1927), ein Artikel zu «Psychotechnik und Psychanalyse» (Schneider, 1921/1922), ein Abdruck aus der Imago ([Imago], 1912/1913), ein Beitrag über «Psychoanalytische Beobachtungen in Alltagsgespräch und Lektüre» (Pye, 1920/1921) oder die Meldung, dass eine "Arbeitsgemeinschaft» geründet worden sei, deren «Hauptinteresse» "auf die psychoanalytische Pädagogik gerichtet» sei (Graber, 1927/1928b, S. 375).

Neben dieser «Arbeitsgemeinschaft» beschäftigen sich mit Psychoanalyse und Pädagogik - immer nach dem Titel geurteilt - 13 Abhandlungen, was knapp 2\% aller Artikel entspricht. Einhellig geht man davon aus, dass die Psychoanalyse einen «intensive[] Wertzuwachs» für die Pädagogik bedeute, weil sie «viel tiefere Blicke in die Zöglingsseele verschaff[en]» könne, wie Pfister in «Die Ergebnisse der Psychanalyse für die Pädagogik» betont ${ }^{9}$. Dasselbe gelte auch für die Seele der Erzieher: "Wollen wir [...] die Erziehung der Jugend reformieren, so weiss ich daher kein besseres Mittel, als dass wir Lehrer uns der Psychanalyse unterziehen» (Pfister, 1913/1914b, S. 243, 244, 251) ${ }^{10}$. Wie ein Artikel über die "Diskussion[en]" im Sommer 1912 in der Psychoanalytischen Vereinigung Zürich zeigt, ist man sich schon früh einig, dass die «innere Beziehung (Uebertragung)" zwischen «Zögling» und «Erzieher» "einer der stärksten Hebel» sei ([Psychoanalytische Vereinigung Zürich], 1912/1913, S. 290, 294-301, 332-333). Die Psychoanalyse hat jedoch nicht nur den Vorteil, "eine wesentliche Vertiefung der Kenntnisse dieser Beziehung herbeigeführt» zu haben ([Psychoanalytische Vereinigung Zürich], 1912/1913, S. 294), sondern erlaubt es natürlich auch, Schülerinnen und Schüler mit Schwierigkeiten zu helfen. Mögliche Schulprobleme gibt es, wie Pfister in «Die Objekte der analytischen Erziehung» ausführt, «unermeßlich viele». Ob es die «völlige Verwirrung» sei, in die "ein zur Wandtafel Gerufener aufs heftigste» gerate, der «förmliche[] intellektuelle[] Heißhunger», mit der ein «Rechner» «die Zahlen verschling[e]», «verräterische[] Verspreche[r]», die gemacht würden, «die zahllosen Arbeiten, in denen ein Schüler seinen alles zersetzenden Pessimismus» ausspreche oder den «Fällen» «im Turnen», «die dem Schwindel unterworfen» seien, stets verspreche die Psychoanalyse Hilfe: Sie sei «unentbehrlich, weil in gewissen Fällen sie allein auf die unterschwelligen Seelenkräfte den erforderlichen Einfluß gewinnen kann» (Pfister, 1918/1919b, S. 263, 266, 267, 268). 
Wie Pfister es in den «Voten» der Psychoanalytischen Vereinigung Zürich zusammenfasst, sind also «zahlreiche Erscheinungen des sittlichen Lebens, die man gewöhnlich als Ausdruck der Bosheit betrachtet, [...] in Wirklichkeit als neurotisch, als Ergebnis einer Triebverklemmung zu betrachten und zu behandeln» ([Psychoanalytische Vereinigung Zürich], 1912/1913, S. 289, 290). Wie der Berner Psychiater Ewald Jung, ein Vetter C. G. Jungs, in «Psychanalyse und Erziehung: Eine kurze Orientierung» hervorhebt, kann «der Erzieher» also dank der Psychoanalyse sowohl durch "seine aktive Mithülfe» "Prophylaxe» betreiben, «durch sinngemäße Beeinflussung» "zur Rettung gefährdeter Kinder» schreiten, als auch kranken Kindern durch die Kur ihre "Gesundheit wieder [...] geben» (Jung, 1921/1922, S. 10-12) ${ }^{11}$.

\section{"Wesen und Aufgaben der psychanalytischen Erziehung"}

Im Artikel «Wesen und Aufgaben der psychanalytischen Erziehung» umreisst Pfister als «das erste Ziel» der Psychoanalyse die "Erlösung aus schädlichen Bindungen»: "Der rein negative Erlösungsbegriff genügt uns jedoch nicht» (Pfister, 1918/1919c, S. 292). Wie Pfister auch schon in den Debatten der Psychoanalytischen Vereinigung Zürich hervorhebt, strebt er eine "positive Ergänzung des negativen analytischen Prozesses» an:

Der Analytiker sollte nur die unter die Bewusstseinsschwelle verdrängten Erinnerungen aufdecken und zur affektvollen Aussprache bringen; um die neuen Bahnen, welche der also befreite Trieb einschlüge, um die «Kompensationen" glaubte er sich nicht bekümmern zu sollen. Dem gegenüber forderte ich von Anfang an Regelung dieser neuen Kanalisation der Triebe durch den Analytiker, weil der Patient, sich selbst überlassen, leicht in neue, unvorhergesehene Verwicklungen gerät und eine dynamische Gesundung niemals mit ethischer Schädigung erkauft werden darf. Ersatz der krankhaften Triebvergeudung durch sittlich-religiös möglichst hohe Willensbestätigung schien mir von Anfang an das Ziel des Analytikers zu sein ([Psychoanalytische Vereinigung Zürich], 1912/1913, S. 289).

Dank dieser «sittlich-religiös[en]», "positive[n] Ergänzung», dieser «neuen Kanalisation der Triebe», könnten z. B. «jugendliche Anarchisten, Pfaffenfresser, Gesinnungslumpen, Weiberhasser, Apostel einer brutalen freien Liebe, Weltverächter, Welteroberer und ähnliche schwierige Klienten» «vor qualvollen, oft lebenslang anhaltenden schweren Irrungen» bewahrt werden (Pfister, 1913/1914b, S. 253). Und da gerade der Erlöser sowohl «Vergangenheit und Gegenwart (Abreaktion und Übertragung)" als "auch die Zukunft, die er kräftig zu erfassen anleitet», «berücksichtigt», sei es «nicht absurd, wenn man die Grundzüge der Pädanalyse bei Jesus vorgezeichnet findet» (Pfister, 1913/1914b, S. 345). 
Die «Pädanalyse» - es handelt sich um Pfisters eigene Wortschöpfung scheint ihren Namen also aus guten Gründen zu tragen. Schliesslich werden «leichte[] Fälle[]» neurotischer Erkrankungen von Schülerinnen und Schülern vom Pädagogen kuriert, der wie ein «Samariter» «erste Hilfe» leistet ([Psychoanalytische Vereinigung Zürich], 1912/1913, S. 290, 328), wobei eben eine besondere, positiv-pädagogische Version der Psychoanalyse zum Einsatz kommt ${ }^{12}$. Diese kuriose Variante, die am «Erbübel» der "Tugend» leide und «das Werk eines zu anständigen Menschen» sei, sowie Pfisters «enthusiastisch verkürzte[] Analysen» kritisiert Freud wiederholt in seinen Briefen an den Pfarrer. Freud versucht Pfister auch geduldig seine «düsteren Theorien» näher zu bringen und betont mehrmals, dass «es nach der Analyse» keiner «Synthese bedarf, vielmehr ist eine gründliche Analyse besonders der Übertragungssituation von Nöten». Denn "wie warmherzig sich der Analytiker auch gebärden mag, er kann es doch nicht übernehmen dem Analysanden Gott und die Vorsehung zu ersetzen» (Freud/Pfister, 1963, S. 36, 121, 126, 130, 144)13.

Die "Pädanalyse», welche die Berner Seminarblätter und die Schulreform propagieren, wird auch vage mit den Bestrebungen der «modernen Pädagogen» verbunden, denen sie helfe, «die Entwicklungsgesetze des Kindes» zu verstehen ([Psychoanalytische Vereinigung Zürich], 1912/1913, S. 293). Auch die «Pädagogik der unbedingten Autoritätsteht im Lichte der Psychanalyse unrühmlich da». So kann man sich dank der Psychoanalyse z. B. gegen Strafen oder für die sexuelle Aufklärung aussprechen (Pfister, 1913/1914b, 256, 297-309). Graber endet seinen Beitrag «Tiefenpsychologische Erziehung» ebenso schwammig wie pathetisch psychoanalytisch-reformpädagogisch:

Durch die harmonische verschränkung jedoch von bewussten und unbewussten tendenzen wird das individuum des werdenden zeitalters mehr und mehr der von aussen kommenden einflüsse entraten können und wird zu grossen taten sich erkühnen, ohne des triebrades der identifikation oder des widerpruches bedürftig zu sein (Graber, 1927/1928a, S. 246)14

Ausschliessliche Fallbeschreibungen verbunden mit theoretischen Ableitungen, wie sie für die zeitgenössische Psychiatrie und die Psychoanalyse selbst typisch sind, gibt es nur zwei: Hans Zulligers Beitrag «Psychoanalyse, Führer und Gemeinschaft» (Zulliger, 1928/1929) und Emmy Niedermanns «Die PsychoAnalyse in der täglichen Erziehung». Niedermann führt jedoch gerade vor, wie «die Analyse, wie wir sie durch C. G. Jung kennen», als erzieherisches "Hilfsmittel» eingesetzt werden kann (Niedermann, 1916/1917, S. 489, 497). Solche theoretische Offenheit oder «ketzerischen» Neigungen zeigen sich auch sonst. In die «Pädagogik der Adlerschen Schule» betont Pfister etwa trotz aller Kritik, dass er viel «von der Adlerschen Psychologie des Selbstbewusstseins gelernt habe», sie einen «eigentümlichen Wert» besitze und er sie nur "warm empfehlen» könne (Pfister, 1914/1915a, S. 161, 171) ${ }^{15}$. Ähnlich irenisch geht der Pfarrer auch mit Jung um (vgl. Pfister 1914/1915b, S. 198-202): 
Die zwischen den verschiedenen psychanalytischen Richtungen [Adler, Jung und Freud] zu Tage tretenden Unterschiede bezeichnen nicht kontradiktorische Gegensätze, sondern zum guten Teil wichtige Ergänzungen, die in ihrer Gesamtheit einen gesunden Fortschritt der neuen für die Kindheitsforschung hochwichtigen Bewegung ausmachen» (Pfister, 1914/1915b, S. 202).

In Wien ist man über eine solche Weitherzigkeit in Theoriefragen natürlich nicht besonders erbaut. So schreibt Freud an Pfister z. B., dass er seiner "Güte, die gerne alles Widerstrebende vereinigen möchte, Zügel» anlegen solle, «sonst läuft wieder alles in Jung'sche Verphrasung aus»; auch erweise er «der Armseligkeit von Adler noch immer zu viel Respekt» (Freud/Pfister, 1963, S. 69, 100). Wie Niedermanns Artikel zeigt, wird eine solche eklektische Position nicht allein von Pfister vertreten. Schneider, der 1912 eine Analyse bei Pfister und 1916 eine bei Jung macht und ebenfalls "eine ziemlich verunreinigte Form von Psychoanalyse» lehrt (Schröter, 2000, S. 25), bietet z. B. im «Sommer-Semester 1918» einen Kurs «Lesen und Besprechen» zu Alfred Adlers und Carl Furtmüllers Heilen und Bilden: Grundlagen der Erziehungskunst für Ärzte und Pädagogen (1913) an ([Arbeitsprogramm], 1918/1919, S. [3]). Und auch in seinem Artikel «Psychotechnik und Psychanalyse» wählt Schneider ein Beispiel aus Jungs Versuch einer Darstellung der psychoanalytischen Theorie (1913). Jungs Vorlesungsreihe wird zwar vor dessen «Trennung» 1914 von Freud publiziert, in einem Artikel 1921 zu Psychoanalyse Freud aber nicht zu erwähnen und stattdessen ein jungsches Beispiel zu wählen (vgl. Schneider, 1921/1922, S. 97), dürfte zumindest «orthodoxen» Leserinnen und Leser sauer aufgestossen sein. Die Schulreform ist also nicht besonders «freudsch" geprägt. Wie es in einer Besprechung von Jungs Psychologischen Typen (1921) heisst: «Freud-Adler-Jung in diesen drei ragenden Gestalten verkörpern sich die heutigen drei ausgeprägten Denkrichtungen der psychanalytischen Wissenschaft» (Zimmermann, 1921/1922, S. 79).

In den zwanziger Jahren tritt die Psychoanalyse bekanntlich ihren internationalen Siegeszug in unzähligen Fachrichtungen sowie im Feuilleton, in der Kunst und im Kino an. Je grösser das Interesse an der Psychoanalyse wird, umso weniger scheint man sich in der Schulreform darum zu kümmern. 1926 scheiden Pfister und Schneider als Herausgeber aus. Ihre letzten psychoanalytischen Beiträge finden sich in den Jahrgängen 1918/1919 und 1921/1922. Obwohl man psychoanalytische Zeitschriften durchaus rezensiert, wird etwa die von Schneider mitherausgegebene Zeitschrift für psychoanalytische Pädagogik, die 1926 zu erscheinen beginnt, in der Schulreform nicht angezeigt. Ganz zum «Schluss», am Ende des letzten Jahrgangs, betont einer der beiden Herausgeber, der Sekundarlehrer und spätere Berner Politiker Fritz Schwarz, der bei Schneider das Seminar besucht hat, die Schulreform sei «oft der letzte Zufluchtsort für Stürmer und Dränger" gewesen: «Sie hat als erste schweizerische Zeitschrift der Kunsterziehungsbewegung, später der Psychanalyse, 1919 den Bestrebungen von Paul Hulligers Schriftreform Raum gewährt» (Schwarz, 1929/1930, S. 360). 
Psychoanalyse scheint also nur eine unter verschiedenen «Schulreformen", die verfolgt werden, und offenbar schon länger wieder passé zu sein.

Obwohl zwei prominente psychoanalytische Pädagogen während über zehn Jahren als Herausgeber der Zeitschrift fungieren, bleibt Psychoanalyse in den Berner Seminarblättern und der Schulreform eine von Pfister dominierte, eklektische und oberflächlich rezipierte Hilfswissenschaft, der keine grössere Beachtung geschenkt wird. Ihre pädagogische Bedeutung wird vor allem darin gesehen, dass sie zu einem besseren psychologischen Verständnis führe und eine "sittlich-religiöse» pädagogische «Erste Hilfe» bei leichten neurotischen «Verletzungen» von Schulkindern erlaube. Verglichen etwa mit den klinischen und theoretischen Bemühungen, die sich in den zur selben Zeit erscheinenden Periodika Imago oder der Internationalen Zeitschrift für ärztliche Psychoanalyse finden, ist der schulreformerische «Berner» Ansatz eher dürftig und der Beitrag "den Streit um die Psychoanalyse von der pädagogischen Praxis aus zu einer [...] positiven Abklärung zu führen», wie Schneider es sich "Zum VI. Jahrgang» wünscht ([Schneider], 1912/1913, 1), bescheiden. An den Berner Seminarblättern und der Schulreform lässt sich daher rückblickend wie im Brennglas der «widersprüchlich[e] und selektiv[e]» Umgang mit Psychoanalyse studieren, der sowohl die Reformpädagogik als auch die psychoanalytische Pädagogik selbst zu Beginn des 20. Jahrhunderts prägt: "Rezeption'» ist immer Bestätigung, nicht kritische Auseinandersetzung» (Oelkers, 2004, S. 794, 796-797). «Freuds Kulturtheorie» kommt dabei «ebenso wenig zum Zuge wie die düsteren Passagen der Theorie des Unbewussten oder des Aggressionstriebes. Erziehung ist ja bei Freud eigentlich eine Grösse, die unmöglich erfolgreich sein kann» (Oelkers, 2002, S. 22). Für «die Schulreformer» wie für die psychoanalytischen Pädagogen hingegen muss «die neue Tiefenpsychologie» vor allem «den pädagogischen Optimismus bestätigen» (Oelkers, 2005, S. 298-299).

\section{Anmerkungen}

1 Als erste wissenschaftliche Neuerung nennt Édouard Claparède "Eugénique», als zweite "Etude et calcul des corrélations». Nach der Psychoanalyse folgen dann «Tests Binet-Simon», "Laboratoire-école», "Instituts pédologiques», "Taylorisme» (Claparède, 1914, S. 248).

2 Zur Gründung des Institut Jean-Jacques Rousseau, zu Théodore Flournoys und Claparèdes Werdegang, der ab 1904 das Genfer Laboratoire pour la psychologie expérimentale leitet, vgl. Hofstetter, 2010, S. 92-203.

3 Vom Unterricht, der zwischen 1912 und 1929 am Institut Jean-Jacques Rousseau erteilt wird, entfallen rund 4\% - 50 Veranstaltungen - auf Psychoanalyse (Hofstetter, 2010, S. 301).

4 Diesen «libéralisme genevois» führt Jean Piaget in seinem Eröffnungsvortrag der Tagung der Schweizerischen Gesellschaft für Psychologie auch gleich selbst vor: Er geht auf Carl Gustav Jung ein (Piaget, 1944, S. 100, 101-102).

5 Zu Ernst Schneider, den Mitarbeitern der Berner Seminarblätter und der Schulreform sowie überhaupt zur Geschichte der psychoanalytischen Pädagogik zu Beginn des 20. Jahrhunderts in der Schweiz vgl. Weber, 1999. 
6 Friedrich Wilhelm Foerster ist zu dieser Zeit Privatdozent für Philosophie in Zürich. Er gibt übrigens die «Vorlage» für den Lehrer August Lindner im Mann ohne Eigenschaften ab. Robert Musil gibt «eine meisterhafte physiognomische Beschreibung» Foersters und "collagiert[]» «ungeniert ganze Kapitel» aus dessen Schriften (Corino, 2003, S. 906).

71909 erscheint Pfisters Religionspädagogisches Neuland: Eine Untersuchung über das Erlebnis- und Arbeitsprinzip im Religionsunterricht (Pfister, 1909a).

8 Auch Oskar Pfister, Eugen Bleuler oder Carl Gustav Jung verwenden den Begriff «Psychanalyse». Pfister zufolge soll es sich beim Kompositum mit «o» um eine falsche Ableitung aus dem Griechischen handeln (Pfister, 1913, S. 16).

9 Zum Argument, dass die Psychoanalyse ein besseres Verständnis der Kinder erlaube, vgl. Jung, 1921/1922, S. 10; [Psychoanalytische Vereinigung Zürich], 1912/1913, S. 293, 303, 322; Schneider, 1915/1916, S. 464.

10 Vgl. Jung 1921/1922, S. 12-13; [Psychoanalytische Vereinigung Zürich], 1912/1913, S. 294-303, 322-323, 332-333.

11 Vgl. Pfister, 19131914b, S. 245; [Psychoanalytische Vereinigung Zürich], 1912/1913, S. 290, 304-308, 322-323.

12 Vgl. Jung, 1921/1922, S. 14.

13 Zur heftigen und schon sehr früh geübten Kritik an Oskar Pfister sowie zu dessen «höchst eigenwillig[er]» (Noth, 2010, S. 101) Psychoanalyse-Rezeption vgl. Noth, 2010, S. 94-108.

14 Zwischen 1926 und 1929 verwendet die Schulreform Kleinschreibung und eine AntiquaSchrift, davor kommt Grossschreibung und Fraktur zum Einsatz, danach für den letzten Jahrgang Grossschreibung und Antiqua.

15 Vgl. auch Pfister, 1913/1914b, S. 298-299.

\section{Literatur}

Anonym (1916/1917). Zum X. Jahr. Die Schulreform, 10, 1.

Arbeitsprogramm (1918/1919). Arbeits-Programm für das Sommer-Semester 1918. Die Schulreform, 12, S. 3-6.

Benetka, G. (1995). Psychologie in Wien. Sozial- und Theoriegeschichte des Wiener Psychologischen Instituts 1922-1933. Wien: WUV-Universitätsverlag.

Bleuler, E. (1926/1927). Zu Siegmund [sic!] Freuds siebzigstem Geburtstag. Die Schulreform, 20, S. 6-12.

Blum, E. (1921/1922). Neurose, Psychanalyse, Christentum. Die Schulreform, 15, S. 266-270.

Bovet, P. (1920). La psychanalyse et l'éducation. Annuaire de l'instruction publique en Suisse, 11, S. 9-38.

Bühler, C. (1922/1923). Das Seelenleben des Jugendlichen. Versuch einer Analyse und Theorie der psychischen Pubertät. Jena: Fischer.

Bühler, P. (2011). Ein «unmöglicher Beruf» - Psychoanalytische Pädagogik zu Beginn des 20. Jahrhunderts. Jahrbuch für historische Bildungsforschung, 17, S. 34-50.

Burri, H. (1928/1929). Psychoanalyse und christlicher Glaube. Die Schulreform, 22, S. 251-276, 356-368, 379-391.

Cifali, M. (2005). L'âme enfantine et la psychanalyse: Entre présent et passé. In M. Ruchat \& C. Magnin, (Hrsg.), "Je suis celui qu'on ne connait pas et qui passe»: Charles Baudouin 1893-1963 (S. 89-105). Lausanne: LEP Loisirs et Pédagogie.

Cifali, M. \& Moll, J. (1995). Zur Begegnung zwischen Pädagogik und Psychoanalyse in Frankreich und in der romanischen Schweiz. Jahrbuch für Psychoanalytische Pädagogik, 7, S. 63-71.

Claparède, E. (1914). Les innovations les plus importantes du domaine de la pédagogie depuis le début du siècle. Jahrbuch der Schweizerischen Gesellschaft für Schulgesundheitspflege, 15, S. 247-256. 
Claparède, E. (1920). Introduction. La Revue de Genève, 1, S. 846-864.

Corino, K. (2003). Robert Musil. Eine Biographie. Reinbek bei Hamburg: Rowohlt.

Foerster, F. W. (1909). Psychoanalyse und Seelsorge. Evangelische Freiheit, 9, S. 335-346, 374-388.

Foerster, F. W. (1910). Nochmals Psychoanalyse und Seelsorge. Evangelische Freiheit, 10, S. 263-275.

Freud, S. \& Pfister, O. (1963). Briefe 1909-1939. Frankfurt am Main: Fischer.

Graber, G. H. (1923/1924). Sigmund Freud und Oswald Spengler. Die Schulreform, 17, S. 368-371.

Graber, G. H. (1927/1928a). Tiefenpsychologische Erziehung. Die Schulreform, 21, S. 243-246.

Graber, G. H. (1927/1928b). Psychoanalytische Arbeitsgemeinschaft. Die Schulreform, 21, S. $375-377$.

Grüninger, U. (1921/1922a). Eine Kritik der Psychanalyse. Die Schulreform, 15, S. 29-37.

Grüninger, U. (1921/1922b). Zur Kritik der Psychanalyse. Eine Entgegnung auf Privatdozent Dr. J. Suter. Die Schulreform, 16, S. 216-220.

Hofstetter, R. (2010). Genève: creuset des sciences de l'éducation (fin du XIX'e siècle - première moitié du XXe siècle). Genève: Droz.

[Imago] (1912/1913). Entwicklung und Ansprüche der Psychoanalyse. Berner Seminarblätter, 6, S. 309-315, 337-346.

Jung, E. (1921/1922). Psychanalyse und Erziehung. Eine kurze Orientierung. Die Schulreform, 15, S. $10-14$

Kohler, R. (2009). Piaget und die Pädagogik. Eine historiographische Analyse. Bad Heilbrunn: Klinkhardt.

Messmer, O. (1911/1912). Die Psychanalyse und ihre pädagogische Bedeutung. Berner Seminarblätter, 5, S. 283-292.

Messmer, O. (1912/1913). Die Psychanalyse in ihrer Entwicklung. Berner Seminarblätter, 6, S. 367-378, 397-408, 417-429, 481-500, 513-520.

Moll, J. (1989). La pédagogie psychanalytique. Origine et histoire. Paris: Dunod.

Niedermann, E. (1916/1917). Die Psycho-Analyse in der täglichen Erziehung. Die Schulreform, 10, S. 489-497.

Noth, I. (2010). Freuds bleibende Aktualität. Psychoanalyserezeption in der Pastoral- und Religionspsychologie im deutschen Sprachraum und in den USA. Stuttgart: Kohlhammer.

Oberholzer, E. (1920). Schweizerische Gesellschaft für Psychoanalyse. Internationale Zeitschrift für Psychoanalyse, 6, S. 101-110.

Oberholzer, E. (1921). Schweizerische Gesellschaft für Psychoanalyse. Internationale Zeitschrift für Psychoanalyse, 7, S. 123-132.

Oelkers, J. (2002). Einige historische Erfahrungen im Verhältnis von Psychologie und Pädagogik. In R. Reichenbach \& F. Oser, (Hrsg.), Die Psychologisierung der Pädagogik. Übel, Notwendigkeit oder Fehldiagnose (S. 12-28). Weinheim, München: Juventa.

Oelkers, J. (2004). Reformpädagogik. In D. Benner \& J. Oelkers (Hrsg.), Historisches Wörterbuch der Pädagogik (S. 783-806). Weinheim, Basel: Beltz.

Oelkers, J. (2005). Reformpädagogik. Eine kritische Dogmengeschichte. Weinheim, München: Juventa.

Ohayon, A. (2006). Psychologie et psychanalyse en France. L'impossible rencontre (1919-1969). Paris: La Découverte.

Pfister, O. (1909a). Religionspädagogisches Neuland. Eine Untersuchung über das Erlebnis- und Arbeitsprinzip im Religionsunterricht. Zürich: Schulthess.

Pfister, O. (1909b). Ein Fall von psychanalytischer Seelsorge und Seelenheilung. Evangelische Freiheit, 9, S. 108-114, 139-149, 175-189. 
Pfister, O. (1910). Die Psychanalyse als wissenschaftliches Prinzip und seelsorgerliche Methode. Evangelische Freiheit, 10, S. 66-73, 102-113, 137-146, 190-200.

Pfister, O. (1913). Die psychanalytische Methode. Leipzig, Berlin: Klinkhardt.

Pfister, O. (1913/1914a). Ernst Dürrs Stellung zur Psychoanalyse. Berner Seminarblätter, 7, S. 289-295.

Pfister, O. (1913/1914b). Die Ergebnisse der Psychanalyse für die Pädagogik. Berner Seminarblätter, 7, S. 241-256, 297-309, 337-347.

Pfister, O. (1914/1915a). Die Pädagogik der Adlerschen Schule. Berner Seminarblätter, 8, S. 159-171.

Pfister, O. (1914/1915b). Psychanalyse und Jugendforschung. Berner Seminarblätter, 8, S. 190-203.

Pfister, O. (1918/1919a). F. W. Foerster - ein Psychanalytiker? Die Schulreform, 12, S. 7-16, $31-46,68-72$.

Pfister, O. (1918/1919b). Die Objekte der analytischen Erziehung. Die Schulreform, 12, S. 263-272.

Pfister, O. (1918/1919c). Wesen und Aufgaben der psychanalytischen Erziehung. Die Schulreform, 12, S. 291-302.

Pfister, O. (1927). In E. Hahn, (Hrsg.), Die Pädagogik der Gegenwart in Selbstdarstellungen. (2. Band, S. 1-47). Leipzig: Meiner.

Piaget, J. (1993). Drei frühe Schriften zur Psychoanalyse. Freiburg im Breisgau: Kore.

Piaget, J. (1944). L'Organisation et l'Esprit de la Psychologie à Genève. Schweizerische Zeitschrift für Psychologie, 3, S. 97-104.

Psychoanalytische Vereinigung Zürich (1912/1913): Psychoanalyse und Pädagogik. Berner Seminarblätter, 6, S. 290-309, 321-337, 366-367.

Pye, P. (1920/1921). Psychoanalytische Beobachtungen in Alltagsgespräch und Lektüre. Die Schulreform, 14, S. 266-269.

Roudinesco, E. (1986). La bataille de cent ans. Histoire de la psychanalyse en France 1885-1939 (1. Band). Paris: Seuil.

Ruchat, M. (2005). Charles Baudouin pédagogue: Un homme entre plusieurs mondes 1915-1933. In M. Ruchat \& C. Magnin (Hrsg.), "Je suis celui qu’on ne connaît pas et qui passe»: Charles Baudouin 1893-1963 (S. 71-88). Lausanne: LEP Loisirs et Pédagogie.

Schneider, E. (1907/1908). Zur Einführung. Berner Seminarblätter, 1, S. 1-3.

Schneider, E. (1908/1909). Zum zweiten Jahr. Berner Seminarblätter, 2, S. 1-2.

Schneider, E. (1912/1913). Zum VI. Jahrgang. Berner Seminarblätter, 6, S. 1.

Schneider, E. (1915/1916). Die Psychanalyse im Psychologieunterricht am Seminar. Berner Seminarblätter, 9, S. 459-464.

Schneider, E. (1921/1922). Psychotechnik und Psychanalyse. Die Schulreform, 15, S. 91-100.

Schneider, E. (1930). Psychoanalyse und Pädagogik. Langensalza: Beyer.

Schröter, M. (2000). Gustav Hans Graber und seine Aufnahme in die DPG, oder: Zum Professionalisierungsstand der deutschen Psychoanalyse um 1930 (mit Dokumenten). Luzifer-Amor, 13, (26), S. 16-41.

Schwarz, F. (1926/1927). Ist eine Analyse ohne Analytiker möglich? Die Schulreform, 20, S. 12-16.

Schwarz, F. (1929/1930). Zum Schluss des Jahrgangs. Die Schulreform, 23, S. 360.

Stern, W. (1913/1914). Die Anwendung der Psychoanalyse auf Kindheit und Jugend. Ein Protest. Mit einem Anhang: Kritik einer Freudschen Kindes-Psychoanalyse von Clara und William Stern. Zeitschrift für angewandte Psychologie und psychologische Sammelforschung, 8, S. 71-101.

Weber, K. (1999): «Es geht ein mächtiges Sehnen durch unsere Zeit!» Reformbestrebungen der Jahrhundertwende und Rezeption der Psychoanalyse am Beispiel der Biographie von Ernst Schneider 1878-1957. Bern: Lang. 
Zimmermann, W. (1921/1922). [Rezension von C. G. Jung: Psychologische Typen]. Die Schulreform, 15, S. 79-81.

Zulliger, H. (1928/1929). Psychoanalyse, Führer und Gemeinschaft. Die Schulreform, 22, S. 346-356.

Schlagworte: Psychoanalyse-Rezeption, Reformpädagogik, Schweiz, Beginn des 20. Jahrhundert.

\section{Le "dernier refuge des Stürmer und Dränger»? La pédagogie psychanalytique dans les Berner Seminarblättern et la Schulreform 1907-1930.}

\section{Résumé}

Les Berner Seminarblätter (appelées plus tard Die Schulreform), une revue dévolue à l'Éducation nouvelle, fut éditée par deux acteurs majeurs de la pédagogie psychanalytique en Suisse allemande: Ernst Schneider et Oskar Pfister. Dans ce périodique de Suisse alémanique, nous pouvons étudier une réception étonamment précoce et positive de la psychanalyse dans le champs pédagogique. Néanmoins et comme cette étude le montre, la psychanalyse fut réceptionnée de manière sélective et sans intérêts particuliers.

Mots-clés: Réception de la psychanalyse, Éducation nouvelle, Suisse, premier $20^{\mathrm{e}}$ siècle.

\section{"L'ultimo rifugio dei Stürmer und Dränger?» La pedagogia psicanalitica nei Berner Seminarblättern e nella Schulreform 1907-1930.}

\section{Riassunto}

La Berner Seminarblättern (più tardi rinominata Die Schulreform), rivista dedicata alla Educazione Nuova, fu curata da due dei maggiori protagonisti della pedagogia psicanalitica in Svizzera tedesca: Ernst Schneider e Oskar Pfister. In questo periodico svizzero-tedesco si rileva una ricezione sorprendentemente precoce e positiva della psicanalisi nel campo della pedagogia. Tuttavia, come dimostra il presente studio, la psicanalisi fu recepita in questo periodico in maniera selettiva e giocoò un ruolo marginale nelle riforme educative dell'epoca.

Parole chiave: Ricezione della psicanalisi, Educazione Nuova, Svizzera, prima metà del $20^{\circ}$ secolo. 


\section{The "last resort of the Stürmer und Dränger»?}

Psychoanalytical education in the Berner Seminarblätter and the Schulreform 1907-1930.

\section{Abstract}

The Berner Seminarblätter, later called Die Schulreform, a journal dedicated to Progressive Education, were edited by two influential psychoanalytical pedagogues: Ernst Schneider and Oskar Pfister. In this Swiss-German periodical an astonishingly early and positive reception of psychoanalysis can be found. But as the article shows in this periodical, of new education, psychoanalysis was taken up very selectively and without particular interest.

Keywords: Reception of psychoanalysis, progressive education, Switzerland, beginning of the $20^{\text {th }}$ century. 
